\title{
Comparaison des méthodes de détermination de la matière grasse dans les tourteaux d'oléagineux - Étude collaborative inter-laboratoires
}

\author{
Alain Quinsac ${ }^{1, a}$, Charles-Pierre Bazin de Caix ${ }^{2}$, Claude Bernard $^{3}$, Brigitte Brosson ${ }^{4}$, Patrick Carre ${ }^{5}$, \\ Gwenaëlle Chardon ${ }^{6}$, Michel Chapron ${ }^{7}$, Danièle Chataignier ${ }^{8}$, Christophe Ferey ${ }^{9}$, Clémence Gaucher ${ }^{2}$, \\ Christophe Genouel ${ }^{10}$, Mohammed Krouti ${ }^{11}$, Renaud Le Bouquin ${ }^{12}$, Jean-Philippe Loison ${ }^{1}$, Hugues \\ Marescot $^{10}$, Valérie Salvador ${ }^{13}$, Ludovic Sarcher ${ }^{9}$, Nathalie Scorze ${ }^{14}$ et Danièle Simonneau ${ }^{15}$ \\ 1 CETIOM, 11 rue Monge Parc Industriel, 33600 Pessac, France \\ 2 AFNOR, 11 rue Francis de Pressensé, 93571 La Plaine Saint Denis, France \\ 3 UNION IN VIVO - Ets INZO, rue de L'Eglise, BP 19 Chierry, 02402 Château-Thierry Cedex, France \\ 4 SAIPOL, Place des Curins, 63190 Lezoux, France \\ 5 CREOL, 11 rue Monge Parc Industriel, 33600 Pessac, France \\ ${ }^{6}$ CARGILL France SAS, rue de Yokosuka, 29200 Brest, France \\ 7 SIFCO, 118 avenue Achille Peretti, 92200 Neuilly sur Seine, France \\ 8 SAIPOL, quai Alfred de Vial, 33530 Bassens, France \\ 9 DELTAVIT SAS, ZA Nord Est du Bois de Teillay, 35150 Janzé, France \\ 10 SCL, 26 avenue Antoine Joly, 35000 Rennes, France \\ 11 CETIOM, 270 avenue de la Pomme de Pin, 45160 Ardon, France \\ 12 LAREAL - EVIALIS, BP 234, 56006 Vannes Cedex, France \\ 13 IDAC, route de Gachet, La Chantrerie, BP 52703, 44327 Nantes Cedex 03, France \\ 14 CCVE SAS, route de Suippes, BP 179, 51009 Chalons en Champagne Cedex, France \\ 15 ARVALIS Institut du Végétal, Station Expérimentale, 91720 Boigneville, France
}

Reçu le 23 janvier 2013 - Accepté le 8 avril 2013

Résumé - Les données recueillies au cours d'une enquête menée sur le long terme en France pour le suivi de la qualité des tourteaux d'oléagineux ont montré que l'utilisation de méthodes d'analyse inadaptées pouvait conduire à une sous-estimation des teneurs en huile résiduelle des tourteaux de colza et de tournesol. Dans le but d'évaluer les performances des méthodes d'analyses actuellement utilisées, une étude collaborative a été réalisée dans le cadre de la commission de normalisation française dans le domaine des aliments des animaux (AFNOR V18A) avec la plupart des laboratoires impliqués dans l'analyse des tourteaux d'oléagineux et des aliments pour animaux. Le circuit d'analyse comprenait huit échantillons, dont cinq avaient une teneur en huile résiduelle faible : trois tourteaux de colza fabriqués dans différentes usines, un tourteau de tournesol, un tourteau de soja. Les trois autres échantillons avaient une teneur en huile élevée et étaient constitués par un extrudat de graines de soja non déshuilé, un tourteau de colza et un tourteau de lin issus de pressage à froid. Les méthodes testées étaient basées sur trois principes : (A) extraction par solvant sans hydrolyse, (B) hydrolyse et extraction par solvant, (C) extraction par solvant sans hydrolyse et avec l'utilisation d'un broyeur à billes. L'étude montre que les données de répétabilité et de reproductibilité obtenues sont en accord avec les résultats de circuits d'analyse réalisés antérieurement et avec les données de fidélité mentionnées dans les normes NF V18-117 Partie 1 pour la méthode A, NF V18-117 Partie 2 pour la méthode B et ISO 734-2 pour la méthode C. Des différences ont été observées entre les trois méthodes pour les teneurs en huile mesurées, en particulier avec les échantillons qui ont subi des traitements thermiques pendant leur fabrication. Les méthodes $\mathrm{A}, \mathrm{C}$ et $\mathrm{B}$ appliquées aux tourteaux déshuilés, conduisent, dans cet ordre, à des teneurs croissantes. La différence entre les résultats des méthodes $\mathrm{A}$ et $\mathrm{B}$, correspondant à la matière grasse liée, varie de 0,7 (tourteau de soja) à $1,4 \mathrm{~g} / 100 \mathrm{~g}$ (tourteau de colza) et représente environ la moitié de la matière grasse mesurée par la méthode A. En comparaison avec la méthode A, l'extraction de la matière grasse non liée avec la méthode $C$ est supérieure de $0,6 \mathrm{~g} / 100 \mathrm{~g}$, ce qui montre l'aptitude de la méthode $\mathrm{C}$ à extraire la matière grasse difficilement accessible. L'étude montre que les trois méthodes donnent, comme prévu, des résultats différents dépendant des principes des méthodes utilisées et des procédés utilisés pour la

\footnotetext{
a Correspondance : quinsac@cetiom.fr
} 
fabrication des tourteaux. En définitive, le choix de la méthode la plus pertinente pour la détermination de la teneur en huile devrait être fait en fonction de l'utilisation prévue des résultats, pour la formulation des aliments des animaux ou pour l'optimisation du procédé d'extraction dans l'usine de trituration.

Mots clés : Huile résiduelle / extraction / tourteau / normalisation / étude collaborative

\begin{abstract}
Comparison of methods for determination of residual oil in oilseed meals. Collaborative interlaboratory study. The data collected in France through a long-term survey on the quality of oilseed meals indicated that the use of unsuitable analytical methods may underestimate the residual oil content of rapeseed and sunflower meal. This misevaluation could make more difficult the optimizations of the oil extraction process and the formulation of feedstuffs. In order to evaluate the performance of current methods, a study was conducted in collaboration with most of the laboratories involved in the analysis of oilseed meal and feed within AFNOR V18A, the French committee for the standardization for feedstuffs. Eight samples were analysed. Five had a low oil content: rapeseed meals (3) from different oil mills, sunflower meal (1), soybean meal (1). Three had a high oil content: extruded full-fat soybeans (1), rapeseed cake (cold-pressed) (1) and linseed cake (cold-pressed) (1). The methods tested were based on three principles: (A) solvent extraction without hydrolysis (B) hydrolysis and solvent extraction (C) without hydrolysis and solvent extraction with a ball mill. The results showed that repeatability and reproducibility data were in agreement with those determined in previous ring-tests and mentioned in standards: NF V18-117 Part 1 for method A, NF V18-117 Part 2 for method B and ISO 734-2 method for C. Some differences for the determination of the oil content were observed between the three methods, particularly for samples that were heated during their production process. Methods A, C and $\mathrm{B}$ in that order gave increasing contents of residual oil in deoiled oilseed meals. The difference between results from methods A and B corresponding to the bound fat ranged from 0.7 (soybean meal) to $1.4 \mathrm{~g} / 100 \mathrm{~g}$ (rapeseed meal) and represented around one half of the fat content determined by the method A. Recovery of the non linked residual oil was higher with method $C$ than with method A by around $0.6 \mathrm{~g} / 100 \mathrm{~g}$ in rapeseed meal, showing the ability of method $\mathrm{C}$ to extract the entrapped oil. This study showed that the three methods gave, as expected, different results depending on the principle of method and the process used for the meal or cake production. Finally, the choice of the most relevant method for fat determination should depend on how the results are used, for feed formulation or for the optimization of the extraction process.
\end{abstract}

Keywords: Residual oil / extraction / oilseed meal / standardization / collaborative study

\section{Introduction}

Les tourteaux de colza et de tournesol déshuilés produits par l'industrie de la trituration représentent depuis quelques années une source de protéines attractive pour l'alimentation animale en raison de propriétés nutritionnelles intéressantes et surtout d'une réelle disponibilité tout au long de l'année. Dans l'objectif de faire mieux connaître les caractéristiques de ces tourteaux et développer leur utilisation, la filière interprofessionnelle (ONIDOL et CETIOM) a engagé depuis 2003 le suivi de la qualité nutritionnelle et sanitaire de la production industrielle, avec la participation dans une enquête mensuelle de la presque totalité des grandes usines de trituration en France.

Les données recueillies entre 2003 et 2006 ont montré que les caractéristiques générales des tourteaux étaient globalement conformes aux valeurs reportées par les bases de données de l'INRA-AFZ pour l'alimentation animale (INRA, 2004) mais que les valeurs de matière grasse $(\mathrm{MG})$ résiduelle mesurées, étaient systématiquement supérieures, surtout pour le tourteau de colza (Dauguet et al., 2006). L'impact de ces écarts est important et constitue un inconvénient aussi bien pour les producteurs que pour les utilisateurs des tourteaux. Par exemple, l'optimisation du process industriel nécessite de connaître précisément la teneur en huile résiduelle, et notamment la fraction inextractible par le solvant, dite «défaut de broyage », qui dépend directement de l'étape de préparation (aplatissage - cuisson - pressage) précédant l'extraction au solvant (Laisney, 1984). Pour l'alimentation des animaux, la teneur résiduelle en MG dans les tourteaux est, en raison de sa forte valeur énergétique, un élément important qu'il faut connaître précisément pour élaborer des formules.

Plusieurs méthodes normalisées pour l'analyse de la MG sont utilisées à plus ou moins bon escient pour les tourteaux d'oléagineux. La norme NF EN ISO 734 est dédiée en particulier aux tourteaux d'oléagineux, la partie 1 constituant la méthode de référence et la partie 2, la méthode rapide. Cette norme comporte une étape de broyage spécifique permettant un meilleur accès du solvant et une extraction plus complète de la MG de la matrice. Dans le domaine de l'alimentation animale, la norme NF V18-117 (1997), remplacée depuis 2011 par la NF ISO 6492, est pratiquée pour les tourteaux bien que ceux-ci soient exclus du champ d'application. En revanche, le règlement 152/2009 (anciennement : directive 98/64/CE modifiant la directive 71/393/CEE) qui a pour domaine d'application les «aliments pour animaux » peut s'appliquer sous certaines conditions aux tourteaux d'oléagineux. Chacun de ces deux textes normatifs (NF V18-117 et règlement 152/2009) comportent deux parties dont l'une (partie A) a pour principe l'extraction directe de la MG par un solvant et l'autre (partie B), fait intervenir avant cette extraction une étape d'hydrolyse pour libérer préalablement la MG liée à la matrice. L'emploi de certaines de ces méthodes pour la collecte des données des bases INRA-AFZ est reconnue (MorandFehr et Tran, 2001) et pourrait expliquer les écarts observés avec les données de l'enquête ONIDOL-CETIOM (Dauguet et al., 2006). En effet, la méthode «aliments pour animaux» partie B, qui est recommandée notamment pour certains produits lorsqu'ils ont été chauffés, n'est, semble-t-il, pas appliquée systématiquement sur les tourteaux d'oléagineux alors 
que ces produits subissent des traitements hydrothermiques en huilerie. En général, c'est la méthode «aliments pour animaux » partie A, sans hydrolyse, qui est utilisée et celle-ci est susceptible de sous-évaluer la teneur en MG, en raison d'un broyage insuffisant de l'échantillon et de l'absence d'hydrolyse qui pourrait libérer la matière grasse liée à la matrice.

Compte tenu des écarts constatés variables selon le type de tourteau et de méthode effectivement pratiquée, il était devenu nécessaire de vérifier l'adéquation des méthodes d'analyses disponibles. L'objectif est de recommander des outils méthodologiques adaptés aux besoins des triturateurs et des utilisateurs des tourteaux pour en assurer la plus grande valorisation. Nous nous proposons dans cette étude de comparer les principales méthodes utilisées en pratique par les laboratoires sur un ensemble représentatif de produits dérivés d'oléagineux contenant des teneurs en MG diverses. Les méthodes correspondent aux normes décrites précédemment et font appel à l'extraction simple de la MG par un solvant non polaire (hexane ou éther de pétrole) avec ou sans broyage ou hydrolyse préalables. Les tourteaux testés représentent les principaux tourteaux utilisés par l'alimentation animale (colza, tournesol, soja, lin). Enfin, les laboratoires qui ont accepté de participer appartiennent aux différents secteurs concernés : triturateurs, laboratoires indépendants, interprofession, laboratoire officiel et fabricants d'aliments. La plupart participent à la commission AFNOR V18A «aliments pour animaux » qui a promu et supervisé cette étude.

\section{Matériels et méthodes}

\subsection{Laboratoires participants}

L'appel à participation lancé au sein de la commission AFNOR V18A (Aliments pour animaux) a réuni douze laboratoires dont six, du secteur de l'alimentation animale, trois de l'industrie de la trituration, deux de centres techniques interprofessionnels agricoles et un laboratoire officiel (Tab. 1).

\subsection{Choix et préparation des échantillons}

La gamme d'échantillons était constituée de tourteaux industriels déshuilés de colza, tournesol et soja, de tourteaux gras de colza et de lin ainsi que de graines de soja extrudées (Tab. 2). Le tourteau de colza était davantage représenté (trois échantillons) en raison de l'hétérogénéité des teneurs en MG résiduelle et des traitements thermiques pratiqués en usine. Les lots de tourteaux ou graines de départ ont été obtenus auprès de divers fournisseurs ou fabricants à l'occasion d'enquêtes sur la qualité réalisées par le CETIOM, ou expressément pour la présente étude. Chacun des huit lots a été homogénéisé et divisé au laboratoire du CETIOM (Ardon-45) en échantillons pour laboratoire de 50 ou 100 g expédiés aux différents laboratoires participants selon le nombre d'analyses à déterminer (Tab. 3).

\subsection{Méthodes testées}

La détermination de la teneur en matière sèche, nécessaire pour l'expression des teneurs en MG, a été conduite par l'ensemble des participants selon la norme NF V03-921 dont le principe est une dessiccation à $103{ }^{\circ} \mathrm{C}$ pendant $4 \mathrm{~h}$. L'objectif étant d'évaluer les méthodes de détermination de la MG pratiquées, chaque laboratoire a appliqué ses méthodes habituelles qui ont été ensuite classées selon leur principe (Tab. 3) dans les catégories A : méthodes sans hydrolyse, B : méthodes avec hydrolyse et $\mathrm{C}$ : méthodes avec broyage. Les modes opératoires des textes de référence (normes ou directives) pour les méthodes A sont similaires; de même pour les méthodes de type B. En revanche, pour les méthodes de type $\mathrm{C}$ appliquées sur les graines oléagineuses ou les tourteaux, les différences sont notables mais pour chacune, un micro-broyeur à billes a été utilisé :

- NF EN ISO 734-2 (méthode rapide « tourteaux ») : microbroyeur à billes avec solvant $10 \mathrm{~min}+$ extraction solvant $1 \mathrm{~h}$.

- NF ISO 659 (méthode de référence «graines ») : extraction au solvant $4 \mathrm{~h}+$ deux fois (micro-broyeur à billes + extraction $2 \mathrm{~h}$ ).

- AFNOR V03-908 (méthode alternative « graines») : micro-broyeur à billes avec solvant $5 \mathrm{~min}+$ extraction au solvant $3 \mathrm{~h}$.

\subsection{Analyses effectuées par les laboratoires}

Une seule détermination de la matière sèche (MS) a été demandée pour chaque échantillon. En revanche, deux répétitions étaient requises pour la détermination de la MG résiduelle sur chaque échantillon afin de mesurer la répétabilité des méthodes testées.

\subsection{Analyses statistiques}

Les données de fidélité ont été déterminées selon la norme NF ISO 5725-2. Les données ont été classées par catégorie de méthodes $\mathrm{A}, \mathrm{B}$ et $\mathrm{C}$. Les données isolées et aberrantes pour la variabilité intra-laboratoire et inter-laboratoires ont été détectées respectivement par les tests de Cochran et Grubbs. Les données fournies par les laboratoires sous forme de répétition unique ont été conservées pour la détermination de la moyenne et de la reproductibilité. Les données isolées (statistique du test supérieure à la valeur critique à $5 \%$ ) ont été conservées et les données aberrantes (statistique du test supérieure à la valeur critique à $1 \%$ ) ont été éliminées. Les tests sur les différences des méthodes d'analyses testées ont été réalisés en déterminant les probabilités critiques de non différence entre les méthodes d'après les variances de reproductibilité des méthodes et la loi de distribution normale (Woonacott et Woonacott, 1988).

\section{Résultats}

\subsection{Participation et bilan des résultats fournis}

Douze laboratoires ont participé à l'étude. La méthode $\mathrm{A}$ a été pratiquée par dix laboratoires dont deux ont fourni une seule répétition au lieu des deux prévues (Tab. 4). La méthode $\mathrm{B}$ a été pratiquée par neuf laboratoires dont deux ont 
Tableau 1. Liste des 12 laboratoires ayant participé à l'étude collaborative.

\begin{tabular}{lll}
\hline Laboratoire & Adresse & Secteur d'activité \\
\hline ARVALIS - Institut du Végétal & Station Expérimentale, 91720 Boigneville & Centre technique \\
SAIPOL - Lezoux & Place des Curins, 63190 Lezoux & Trituration \\
CARGILL France SAS & Rue de Yokosuka, 29200 Brest & Trituration \\
CCVE SAS & Route de Suippes, BP 179, 51009 Chalons en Champagne cedex & Alimentation animale \\
CETIOM & 270 avenue de la Pomme de Pin, 45160 Ardon & Centre technique \\
DELTAVIT SAS & ZA Nord Est du Bois de Teillay, 35150 Janzé & Alimentation animale \\
IDAC & Route de Gachet, La Chantrerie, BP 52703, 44327 Nantes Cedex 03 & Alimentation animale \\
LAREAL - EVIALIS & BP 234, 56006 Vannes cedex & Alimentation animale \\
SAIPOL - Bassens & Quai Alfred de Vial, 33530 Bassens & Trituration \\
Service Commun des Laboratoires & 26 Avenue Antoine Joly, 35000 Rennes & DGCCRF-DGDDI \\
SIFCO & 118 avenue Achille Peretti, 92200 Neuilly sur Seine & Alimentation animale \\
UNION IN VIVO - Ets INZO & Rue de L'Eglise, BP 19 Chierry, 02402 Château-Thierry cedex & Alimentation animale \\
\hline
\end{tabular}

Tableau 2. Liste des échantillons analysés dans le ring-test.

\begin{tabular}{cll}
\hline Référence & Échantillons & Origine \\
\hline 1 & tourteau colza industriel déshuilé & SAIPOL, Compiègne - 60 \\
2 & tourteau colza industriel déshuilé & SAIPOL, Dieppe - 76 \\
3 & tourteau colza industriel déshuilé & SAIPOL, Lezoux - 63 \\
4 & tourteau de tournesol industriel déshuilé & SAIPOL, Bassens - 33 \\
5 & tourteau soja industriel déshuilé & CARGILL France SAS, Brest - 29 \\
6 & tourteau de colza gras pressé à froid & Pressage à la ferme - 86 \\
7 & graine de soja extrudée & DIALZO, Vergt - 24 \\
8 & tourteau de lin de première pression & Huilerie COACHE, Vitz sur Authie - 80 \\
\hline
\end{tabular}

Tableau 3. Types de méthodes utilisées pour la détermination de la matière grasse résiduelle.

\begin{tabular}{lccc}
\hline Types de méthode & $\mathrm{A}$ & $\mathrm{B}$ & $\mathrm{C}$ \\
\hline \multirow{2}{*}{ Principe } & \multicolumn{2}{c}{ Extraction par solvant apolaire (éther de pétrole ou hexane pendant 4 $\mathrm{h}$ ) } \\
\cline { 2 - 4 } & \multicolumn{2}{c}{ Avec hydrolyse } & Avec broyage \\
\hline \multirow{2}{*}{ Textes de référence } & NF V18-117 partie A & NF V18-117 partie B & NF EN ISO 734-2 (2008) \\
(en 2009) & Directive 71/393/CEE & Directive 71/393/CEE & NF EN ISO 659 (2009) \\
& modifiée partie A & modifiée partie B & AFNOR V03-908 \\
\hline
\end{tabular}

Note : Depuis la fin de l'étude, l'indexation de certains textes de référence a changé. La NF V18-117 a été abrogée en mars 2011 et remplacée par la NF ISO 6492 équivalente. La directive 71/393/CEE modifiée a été abrogée et remplacée en 2009 par le Règlement R152/2009 ANNEXE III totalement équivalent. Par ailleurs, la norme AFNOR V03-908 a été homologuée NF en 2009 et les normes NF EN ISO 659 et NF EN ISO 734-2 ont été révisées, respectivement, en 2009 et 2010.

fourni une seule répétition au lieu des deux prévues (Tab. 5). Enfin, la méthode $\mathrm{C}$ a été pratiquée par sept laboratoires dont trois ont fourni une répétition au lieu des deux prévues (Tab. 6).

\subsection{Validation des données brutes}

Sur un total de 144 données fournies par les laboratoires pour la méthode $\mathrm{A}$, trois ont été éliminées en raison de la variabilité inter-laboratoires par le test de Grubbs. Pour la méthode B, sur un total de 120 données fournies, deux ont été éliminées en raison de la variabilité intra-laboratoire par le test de Cochran. Pour la méthode C, sur un total de 93 données fournies, deux ont été éliminées en raison de la variabilité intra-laboratoire et inter-laboratoires et deux autres en raison de la variabilité inter-laboratoires. Les Tableaux 4-6 indiquent les données conservées et éliminées par l'analyse statistique. Le nombre de laboratoires conservés est 9 ou 10 pour la méthode $\mathrm{A}, 8$ ou 9 pour la méthode $\mathrm{B}$ et de 5 à 7 dans le cas de la méthode $\mathrm{C}$.

\subsection{Répétabilité}

Les résultats du circuit obtenus après traitement des données figurent dans le Tableau 7. Les valeurs des écart-types de répétabilité $\left(s_{r}\right)$ pour la méthode A sont comprises entre 0,05 et $0,07 \mathrm{~g} / 100 \mathrm{~g}$ pour les tourteaux déshuilés et sont voisines de $0,15 \mathrm{~g} / 100 \mathrm{~g}$ MS pour les autres échantillons beaucoup plus riches en $\mathrm{MG}$, ce qui pour ces derniers, représente un coefficient de variation de moins de $1 \%$. Pour les méthodes B et $\mathrm{C}$, les valeurs des écarts-types de répétabilité sont un peu 
Tableau 4. Résultats obtenus avec les méthodes d'extraction sans hydrolyse (méthode A).

\begin{tabular}{|c|c|c|c|c|c|c|c|c|c|c|}
\hline Labo & Méthode & Échantillon & $\begin{array}{c}\text { tx-col-1 } \\
\mathbf{1}\end{array}$ & $\begin{array}{c}\text { tx-col-2 } \\
2\end{array}$ & $\begin{array}{c}\text { tx-col-3 } \\
\mathbf{3}\end{array}$ & $\begin{array}{c}\text { tx-tourn. } \\
4\end{array}$ & $\begin{array}{c}\text { tx-soja } \\
\mathbf{5}\end{array}$ & $\begin{array}{c}\text { colz-exp } \\
\mathbf{6}\end{array}$ & $\begin{array}{c}\text { soja-ext } \\
7\end{array}$ & $\begin{array}{c}\text { lin-exp } \\
\mathbf{8}\end{array}$ \\
\hline \multirow{4}{*}{1} & \multirow{4}{*}{$\begin{array}{l}\text { DIR 98/64/CEE } \\
\text { procédé A }\end{array}$} & $\% \mathrm{MS}$ & 90,90 & 90,40 & 90,40 & 91,50 & 87,50 & 90,30 & 89,70 & 92,20 \\
\hline & & rep 1 & 1,89 & 3,69 & 2,81 & 1,43 & 2,02 & 17,31 & 18,56 & $18,54 * \mathrm{C}$ \\
\hline & & rep 2 & 1,88 & 3,67 & 2,91 & 1,36 & 2,06 & 17,26 & 18,15 & $19,08 * \mathrm{C}$ \\
\hline & & Moy & 1,89 & 3,68 & 2,86 & 1,39 & 2,04 & 17,29 & 18,36 & 18,81 \\
\hline \multirow{4}{*}{2} & \multirow{4}{*}{$\begin{array}{l}\text { DIR 98/64/CEE } \\
\text { procédé A }\end{array}$} & $\% \mathrm{MS}$ & 90,50 & 89,90 & 90,10 & 91,40 & 87,10 & 89,90 & 89,50 & 92,20 \\
\hline & & rep 1 & 1,88 & 4,12 & 2,77 & 5,80 & 2,99 & 17,46 & 18,88 & 18,87 \\
\hline & & rep 2 & - & - & - & - & - & - & - & - \\
\hline & & Moy & 1,88 & 4,12 & 2,77 & $\mathbf{5 , 8 0} * * \mathbf{G}$ & 2,99 & 17,46 & 18,88 & 18,87 \\
\hline \multirow{4}{*}{3} & \multirow{4}{*}{$\begin{array}{l}\text { MGA NF V18-117 } \\
\text { sans hydrolyse }\end{array}$} & $\% \mathrm{MS}$ & 90,72 & 90,18 & 90,44 & 91,29 & 87,84 & 90,44 & 89,86 & 92,06 \\
\hline & & rep 1 & 1,77 & 3,58 & 2,70 & 1,39 & 1,97 & 17,48 & 18,30 & 18,46 \\
\hline & & rep 2 & 1,82 & 3,57 & 2,68 & 1,36 & 1,89 & 17,45 & 18,68 & 18,41 \\
\hline & & Moy & 1,80 & 3,58 & 2,69 & 1,37 & 1,93 & 17,46 & 18,49 & 18,43 \\
\hline \multirow{4}{*}{4} & & $\% \mathrm{MS}$ & 90,60 & 90,10 & 90,40 & 91,40 & 87,70 & 90,10 & 89,80 & 92,00 \\
\hline & NF V18-117 & rep 1 & 1,21 & 3,22 & 1,88 & 1,20 & 1,94 & 16,98 & 16,82 & 18,37 \\
\hline & (Août 1997) & rep 2 & 1,32 & 3,33 & 1,99 & 1,20 & 2,05 & 16,76 & 16,82 & 18,48 \\
\hline & sans ajout acétone & Moy & $1,27 * G$ & 3,27 & $1,94 *$ & 1,20 & 2,00 & 16,87 & $16,82 * * \mathbf{G}$ & 18,42 \\
\hline \multirow{4}{*}{5} & \multirow{4}{*}{$\begin{array}{c}\text { V18-117 } \\
\text { «soxtec } » 1 \mathrm{~h} 30\end{array}$} & $\% \mathrm{MS}$ & 90,40 & 89,90 & 90,30 & 91,50 & 87,50 & 90,00 & 89,60 & 92,00 \\
\hline & & rep 1 & 2,10 & 4,00 & 2,88 & 1,64 & 2,06 & 17,56 & 18,42 & 19,13 \\
\hline & & rep 2 & 1,99 & 3,89 & 2,99 & 1,53 & 2,06 & 17,67 & 18,53 & 18,80 \\
\hline & & Moy & 2,05 & 3,95 & 2,93 & 1,58 & 2,06 & 17,61 & 18,47 & 18,97 \\
\hline \multirow{4}{*}{6} & \multirow{4}{*}{$\begin{array}{c}\text { Directive CE 71/393 } \\
\text { Partie A «Soxtec } » 1 \text { h30 }\end{array}$} & $\% \mathrm{MS}$ & 90,40 & 90,00 & 90,30 & 91,30 & 87,80 & 90,00 & 89,70 & 92,00 \\
\hline & & rep 1 & 1,92 & 3,77 & 2,83 & 1,53 & 2,44 & 17,63 & 18,38 & 18,93 \\
\hline & & rep 2 & 2,01 & 3,82 & 2,88 & 1,53 & 2,57 & 17,47 & 18,63 & 18,97 \\
\hline & & Moy & 1,97 & 3,79 & 2,86 & 1,53 & 2,51 & 17,55 & 18,51 & 18,95 \\
\hline \multirow{4}{*}{7} & \multirow{4}{*}{$\begin{array}{c}\text { Dir CEE 98/64 } \\
\text { proc A Extracteur } \\
\text { automatique }\end{array}$} & $\% \mathrm{MS}$ & 90,64 & 90,06 & 90,44 & 91,55 & 87,56 & 90,00 & 89,73 & 92,20 \\
\hline & & rep 1 & 2,23 & 3,65 & 3,22 & 1,44 & 2,19 & 17,31 & 18,67 & 18,68 \\
\hline & & rep 2 & 2,29 & 3,70 & 3,30 & 1,56 & 2,11 & 17,14 & 18,75 & 18,60 \\
\hline & & Moy & 2,26 & 3,68 & 3,26 & 1,50 & 2,15 & 17,23 & 18,71 & 18,64 \\
\hline \multirow{4}{*}{8} & \multirow{4}{*}{$\begin{array}{l}\text { NF V18-117 } \\
\text { procédé A }\end{array}$} & $\% \mathrm{MS}$ & 90,34 & 89,70 & 90,03 & 91,15 & 87,05 & 89,85 & 89,26 & 91,67 \\
\hline & & rep 1 & 1,66 & 3,68 & 2,67 & 1,43 & 2,18 & 17,14 & 18,71 & 18,76 \\
\hline & & rep 2 & 1,77 & 3,57 & 2,55 & 1,43 & 2,18 & 16,81 & 18,82 & 18,87 \\
\hline & & Moy & 1,72 & 3,62 & 2,61 & 1,43 & 2,18 & 16,97 & 18,77 & 18,82 \\
\hline \multirow{4}{*}{9} & \multirow{4}{*}{$\begin{array}{l}\text { NF V18-117 } \\
\text { procédé A }\end{array}$} & $\% \mathrm{MS}$ & 90,50 & 90,00 & 90,20 & 91,30 & 87,70 & 90,20 & 89,90 & 92,00 \\
\hline & & rep 1 & 1,88 & 3,89 & 2,66 & 1,53 & 2,51 & 17,96 & 18,58 & 19,35 \\
\hline & & rep 2 & 1,88 & 4,00 & 2,66 & 1,75 & 2,51 & 17,74 & 18,58 & 19,35 \\
\hline & & Moy & 1,88 & 3,94 & 2,66 & 1,64 & 2,51 & 17,85 & 18,58 & 19,35 \\
\hline \multirow{4}{*}{10} & \multirow{4}{*}{$\begin{array}{c}\text { NFV-18104 } \\
\text { avec éther de P }\end{array}$} & $\% \mathrm{MS}$ & 90,50 & 89,90 & 90,40 & 91,40 & 87,60 & 90,00 & 89,80 & 92,10 \\
\hline & & rep 1 & 1,99 & 3,78 & 2,88 & 1,31 & 2,40 & 17,22 & 18,49 & 19,54 \\
\hline & & rep 2 & - & - & - & - & - & - & - & - \\
\hline & & Moy & 1,99 & 3,78 & 2,88 & 1,31 & 2,40 & 17,22 & 18,49 & 19,54 \\
\hline \multicolumn{11}{|c|}{ Analyse statistique } \\
\hline \multirow{2}{*}{\multicolumn{2}{|c|}{ Nombre de laboratoires }} & total & 10 & 10 & 10 & 10 & 10 & 10 & 10 & 10 \\
\hline & & conservés & 10 & 10 & 10 & 9 & 10 & 10 & 9 & 10 \\
\hline Nomb & re de données & répétabilité & 16 & 16 & 16 & 16 & 16 & 16 & 14 & 16 \\
\hline conser & vées pour mesure de & reproductibilité & 18 & 18 & 18 & 17 & 18 & 18 & 16 & 18 \\
\hline
\end{tabular}

Note : Les laboratoires $n^{\circ} 11,12$ et 13 n’ont pas pratiqué cette méthode. ( ${ }^{*} \mathrm{C},{ }^{*} \mathrm{C}$ : tests de Cochran, respectivement, valeur isolée conservée ou aberrante écartée. Lorsque les deux répétitions sont aberrantes, les moyennes ne sont pas conservées pour l'analyse statistique.* $\mathrm{G}$, **G : tests de Grubbs, respectivement, valeur isolée conservée ou aberrante écartée). 
Tableau 5. Résultats obtenus avec les méthodes d'extraction avec hydrolyse (méthode B).

\begin{tabular}{|c|c|c|c|c|c|c|c|c|c|c|}
\hline Labo & Méthode & Échantillon & $\begin{array}{c}\text { tx-col-1 } \\
\mathbf{1}\end{array}$ & $\begin{array}{c}\text { tx-col-2 } \\
\mathbf{2}\end{array}$ & $\begin{array}{c}\text { tx-col-3 } \\
\mathbf{3}\end{array}$ & $\begin{array}{c}\text { tx-tourn. } \\
\mathbf{4}\end{array}$ & $\begin{array}{c}\text { tx-soja } \\
\mathbf{5}\end{array}$ & $\begin{array}{c}\text { colz-exp } \\
\mathbf{6}\end{array}$ & $\begin{array}{c}\text { soja-ext } \\
7\end{array}$ & $\begin{array}{c}\text { lin-exp } \\
\mathbf{8}\end{array}$ \\
\hline \multirow{4}{*}{1} & \multirow{4}{*}{$\begin{array}{c}\text { DIR 98/64/CEE } \\
\text { procédé B }\end{array}$} & $\% \mathrm{MS}$ & 90,90 & 90,40 & 90,40 & 91,50 & 87,50 & 90,30 & 89,70 & 92,20 \\
\hline & & rep 1 & 3,82 & 5,07 & $5,76 * * \mathrm{C}$ & 2,54 & $2,89 * \mathrm{C}$ & 18,42 & 22,12 & $18,46^{*} \mathrm{C}$ \\
\hline & & rep 2 & 3,22 & 4,87 & $4,61 * * \mathrm{C}$ & 2,68 & $2,70 * \mathrm{C}$ & 18,52 & 21,96 & $20,03^{*} \mathrm{C}$ \\
\hline & & Moy & 3,52 & 4,97 & $5,19 * * G$ & 2,61 & 2,79 & 18,47 & 22,04 & 19,25 \\
\hline \multirow{4}{*}{2} & \multirow{4}{*}{$\begin{array}{l}\text { DIR 98/64/CEE } \\
\text { procédé B }\end{array}$} & $\% \mathrm{MS}$ & 90,50 & 89,90 & 90,10 & 91,40 & 87,10 & 89,90 & 89,50 & 92,20 \\
\hline & & rep 1 & 3,10 & 4,60 & 4,10 & 2,10 & 2,80 & 16,10 & 19,60 & 17,90 \\
\hline & & rep 2 & - & - & - & - & - & - & - & - \\
\hline & & Moy & 3,10 & 4,60 & 4,10 & 2,10 & 2,80 & 16,10 & 19,60 & 17,90 \\
\hline \multirow{4}{*}{3} & \multirow{4}{*}{$\begin{array}{c}\text { MGA NF V18-117 } \\
\text { avec hydrolyse }\end{array}$} & $\% \mathrm{MS}$ & 90,72 & 90,18 & 90,44 & 91,29 & 87,84 & 90,44 & 89,86 & 92,06 \\
\hline & & rep 1 & 3,32 & 4,73 & 4,10 & 2,29 & 2,80 & 17,91 & 21,24 & 18,78 \\
\hline & & rep 2 & 3,40 & 4,87 & 4,02 & 2,31 & 2,85 & 17,77 & 21,22 & 18,62 \\
\hline & & Moy & 3,36 & 4,80 & 4,06 & 2,30 & 2,82 & 17,84 & 21,23 & 18,70 \\
\hline \multirow{4}{*}{4} & \multirow{4}{*}{$\begin{array}{c}\text { NF V18-117 } \\
\text { (Août 1997) } \\
\text { avec hydrolyse }\end{array}$} & $\% \mathrm{MS}$ & 90,60 & 90,10 & 90,40 & 91,40 & 87,70 & 90,10 & 89,80 & 92,00 \\
\hline & & rep 1 & 3,09 & 4,55 & 4,20 & 2,30 & 3,19 & 16,76 & 21,05 & 18,91 \\
\hline & & rep 2 & 2,65 & 4,55 & 4,09 & 2,19 & 3,31 & 17,65 & 21,05 & 18,91 \\
\hline & & Moy & 2,87 & 4,55 & 4,15 & 2,24 & 3,25 & 17,20 & 21,05 & 18,91 \\
\hline \multirow{4}{*}{5} & \multirow{4}{*}{$\begin{array}{c}\text { V18-117 soxtec } \\
\text { 1h30 avec hydrolyse }\end{array}$} & $\% \mathrm{MS}$ & 90,40 & 89,90 & 90,30 & 91,50 & 87,50 & 90,00 & 89,60 & 92,00 \\
\hline & & rep 1 & 3,32 & 4,67 & $3,77 * \mathrm{C}$ & 2,40 & 2,97 & 17,56 & $20,98 * \mathrm{C}$ & 18,04 \\
\hline & & rep 2 & 3,21 & 4,78 & $4,10 * \mathrm{C}$ & 2,19 & 2,97 & 18,22 & $21,43 * \mathrm{C}$ & 19,13 \\
\hline & & Moy & 3,26 & 4,73 & 3,93 & 2,30 & 2,97 & 17,89 & 21,21 & 18,59 \\
\hline \multirow{4}{*}{6} & \multirow{4}{*}{$\begin{array}{l}\text { Directive CE 71/393 } \\
\text { Partie B Soxtec 1h30 }\end{array}$} & $\% \mathrm{MS}$ & 90,40 & 90,00 & 90,30 & 91,30 & 87,80 & 90,00 & 89,70 & 92,00 \\
\hline & & rep 1 & 3,38 & 4,97 & 4,29 & 2,28 & 2,86 & 17,97 & 20,43 & 19,29 \\
\hline & & rep 2 & 3,35 & 5,11 & 4,13 & 2,31 & 2,87 & 17,91 & 20,19 & 19,38 \\
\hline & & Moy & 3,37 & 5,04 & 4,21 & 2,29 & 2,86 & 17,94 & 20,31 & 19,34 \\
\hline \multirow{4}{*}{7} & \multirow{4}{*}{$\begin{array}{l}\text { Directive CEE 98/64 } \\
\text { procédé B Extracteur } \\
\text { automatique }\end{array}$} & $\% \mathrm{MS}$ & 90,64 & 90,06 & 90,44 & 91,55 & 87,56 & 90,00 & 89,73 & 92,20 \\
\hline & & rep 1 & 2,82 & 5,20 & 4,35 & 2,41 & 2,82 & 17,63 & 21,48 & 19,00 \\
\hline & & rep 2 & 2,60 & 5,13 & 4,31 & 2,41 & 2,88 & 17,76 & 21,44 & 18,79 \\
\hline & & Moy & 2,71 & 5,16 & 4,33 & 2,41 & 2,85 & 17,69 & 21,46 & 18,89 \\
\hline \multirow{4}{*}{8} & \multirow{4}{*}{$\begin{array}{l}\text { NF V18-117 } \\
\text { procédé B }\end{array}$} & $\% \mathrm{MS}$ & 90,34 & 89,70 & 90,03 & 91,15 & 87,05 & 89,85 & 89,26 & 91,67 \\
\hline & & rep 1 & 3,54 & 4,68 & 4,11 & 2,41 & 3,10 & 16,81 & 18,71 & 19,42 \\
\hline & & rep 2 & - & - & - & - & - & - & - & - \\
\hline & & Moy & 3,54 & 4,68 & 4,11 & 2,41 & 3,10 & 16,81 & 18,71 & 19,42 \\
\hline \multirow{4}{*}{10} & \multirow{4}{*}{$\begin{array}{c}\text { NFV-18104 avec hydrolyse } \\
\text { acide et éther } \\
\text { de Pétrole }\end{array}$} & $\% \mathrm{MS}$ & 90,50 & 89,90 & 90,40 & 91,40 & 87,60 & 90,00 & 89,80 & 92,10 \\
\hline & & rep 1 & 3,76 & 4,89 & 4,54 & 2,63 & 3,08 & 17,44 & 21,27 & 19,87 \\
\hline & & rep 2 & - & - & - & - & - & - & - & - \\
\hline & & Moy & 3,76 & 4,89 & 4,54 & 2,63 & 3,08 & 17,44 & 21,27 & 19,87 \\
\hline
\end{tabular}

\begin{tabular}{l|l|cccccccc}
\hline \multicolumn{1}{l|}{ Analyse statistique } & total & 9 & 9 & 8 & 9 & 9 & 9 & 9 & 9 \\
\cline { 2 - 10 } Nombre de laboratoires & conservés & 9 & 9 & 8 & 9 & 9 & 9 & 9 & 9 \\
\hline \multirow{2}{*}{$\begin{array}{l}\text { Nombre de données } \\
\text { conservées pour mesure de }\end{array}$} & répétabilité & 12 & 12 & 10 & 12 & 12 & 12 & 12 & 12 \\
\cline { 2 - 10 } & reproductibilité & 15 & 15 & 13 & 15 & 15 & 15 & 15 & 15 \\
\hline
\end{tabular}

Note : Les laboratoires $n^{\circ} 9,11$ et 12 n'ont pas pratiqué cette méthode. (* $\mathrm{C}$, **C : tests de Cochran, respectivement, valeur isolée conservée ou aberrante écartée. Lorsque les deux répétitions sont aberrantes, les moyennes ne sont pas conservées pour l'analyse statistique.* $\mathrm{G}$, ** $\mathrm{G}$ : tests de Grubbs, respectivement, valeur isolée conservée ou aberrante écartée). 
Tableau 6. Résultats obtenus avec les méthodes d'extraction avec broyage (méthode C).

\begin{tabular}{|c|c|c|c|c|c|c|c|c|c|c|c|}
\hline \multirow{2}{*}{ Labo } & \multirow{2}{*}{\multicolumn{2}{|c|}{ Méthode }} & \multirow{2}{*}{ Échantillon } & tx-col-1 & tx-col-2 & tx-col-3 & tx-tourn. & tx-soja & colz-exp & soja-ext & lin-exp \\
\hline & & & & 1 & 2 & 3 & 4 & 5 & 6 & 7 & 8 \\
\hline \multirow{4}{*}{1} & \multirow{4}{*}{\multicolumn{2}{|c|}{$\begin{array}{c}\text { ISO 734-2 (1 à } 5+8) \\
\text { V03-908 (6 et } 7)\end{array}$}} & $\% \mathrm{MS}$ & 90,90 & 90,40 & 90,40 & 91,50 & 87,50 & 90,30 & 89,70 & 92,20 \\
\hline & & & rep 1 & 1,86 & 3,95 & 3,26 & 1,66 & 2,35 & 18,01 & 19,84 & $18,02 * * \mathrm{C}$ \\
\hline & & & rep 2 & 2,18 & 4,12 & 3,19 & 1,56 & 2,88 & 18,04 & 20,13 & $18,84 * * \mathrm{C}$ \\
\hline & & & Moy & 2,02 & 4,03 & 3,22 & 1,61 & 2,62 & 18,02 & 19,99 & $18,43 * G$ \\
\hline \multirow{4}{*}{3} & \multirow{4}{*}{\multicolumn{2}{|c|}{$\begin{array}{c}\text { Broyage Dangoumau + } \\
\text { procédé A }\end{array}$}} & $\% \mathrm{MS}$ & 90,72 & 90,18 & 90,44 & 91,29 & 87,84 & 90,44 & 89,86 & 92,06 \\
\hline & & & rep 1 & 2,52 & 4,25 & 3,31 & 1,40 & 2,28 & 17,78 & 19,60 & 19,12 \\
\hline & & & rep 2 & 2,56 & 4,19 & 3,48 & 1,67 & 2,31 & 17,41 & 19,79 & 19,24 \\
\hline & & & Moy & 2,54 & 4,22 & 3,39 & 1,53 & 2,29 & 17,60 & 19,69 & 19,18 \\
\hline \multirow{4}{*}{5} & \multirow{4}{*}{\multicolumn{2}{|c|}{$\begin{array}{c}\text { Broyage } \\
\text { Dangoumau }\end{array}$}} & $\% \mathrm{MS}$ & 90,40 & 89,90 & 90,30 & 91,50 & 87,50 & 90,00 & 89,60 & 92,00 \\
\hline & & & rep 1 & - & - & 3,31 & 1,63 & 2,39 & 17,91 & 19,72 & 19,17 \\
\hline & & & rep 2 & - & - & - & - & - & - & - & - \\
\hline & & & Moy & - & - & 3,31 & 1,63 & 2,39 & 17,91 & 19,72 & 19,17 \\
\hline \multirow{4}{*}{8} & \multirow{4}{*}{\multicolumn{2}{|c|}{ V03-908 }} & $\% \mathrm{MS}$ & 90,34 & 89,70 & 90,03 & 91,15 & 87,05 & 89,85 & 89,26 & 91,67 \\
\hline & & & rep 1 & 2,88 & 4,35 & $3,67 * \mathrm{C}$ & 1,97 & 2,64 & 17,81 & 19,49 & $19,42 * \mathrm{C}$ \\
\hline & & & rep 2 & 2,55 & 4,01 & $3,22 * \mathrm{C}$ & 1,54 & 2,99 & 17,47 & 19,72 & $19,09 * \mathrm{C}$ \\
\hline & & & Moy & 2,71 & 4,18 & 3,44 & 1,76 & 2,81 & 17,64 & 19,61 & 19,25 \\
\hline \multirow{4}{*}{9} & \multirow{4}{*}{\multicolumn{2}{|c|}{ ISO $734-2$}} & $\% \mathrm{MS}$ & 90,50 & 90,00 & 90,20 & 91,30 & 87,70 & 90,20 & 89,90 & 92,00 \\
\hline & & & rep 1 & 2,76 & 4,67 & 3,77 & 1,86 & 2,85 & 18,29 & 20,58 & 19,24 \\
\hline & & & rep 2 & 2,87 & 4,56 & 3,77 & 1,75 & 2,85 & 18,29 & 20,80 & 19,24 \\
\hline & & & Moy & 2,82 & 4,61 & 3,77 & 1,81 & 2,85 & 18,29 & 20,69 & 19,24 \\
\hline \multirow{4}{*}{11} & \multirow{4}{*}{\multicolumn{2}{|c|}{ ISO 659}} & $\% \mathrm{MS}$ & 90,66 & 90,13 & 90,41 & 91,47 & 87,73 & 90,23 & 89,89 & 92,51 \\
\hline & & & rep 1 & 2,25 & 4,11 & 3,46 & 1,69 & 2,88 & 17,60 & 20,81 & 18,67 \\
\hline & & & rep 2 & 2,27 & 4,11 & 3,43 & 1,68 & 2,87 & 17,51 & 20,79 & 18,66 \\
\hline & & & Moy & 2,26 & 4,11 & 3,45 & 1,69 & 2,88 & 17,56 & $20,80 * G$ & $18,66 * * G$ \\
\hline \multirow{4}{*}{12} & \multirow{4}{*}{\multicolumn{2}{|c|}{ ISO 659}} & $\% \mathrm{MS}$ & 90,21 & 89,67 & 91,22 & 87,13 & - & 89,76 & 89,39 & 91,90 \\
\hline & & & rep 1 & 2,25 & 4,45 & 3,10 & 1,61 & - & 18,05 & 19,77 & 19,00 \\
\hline & & & rep 2 & - & - & - & - & - & - & - & - \\
\hline & & & Moy & 2,25 & 4,45 & 3,10 & 1,61 & - & 18,05 & 19,77 & 19,00 \\
\hline \multicolumn{12}{|c|}{ Analyse statistique } \\
\hline \multirow{2}{*}{\multicolumn{2}{|c|}{ Nombre de laboratoires }} & tota & & 6 & 6 & 7 & 7 & 6 & 7 & 7 & 7 \\
\hline & & con & servés & 6 & 6 & 7 & 7 & 6 & 7 & 7 & 5 \\
\hline \multirow{2}{*}{\multicolumn{2}{|c|}{$\begin{array}{l}\text { Nombre de données } \\
\text { conservées pour mesure de }\end{array}$}} & rép & Ctabilité & 10 & 10 & 10 & 10 & 10 & 10 & 10 & 6 \\
\hline & & rep & oductibilité & 11 & 11 & 12 & 12 & 11 & 12 & 12 & 8 \\
\hline
\end{tabular}

Note : Les laboratoires $\mathrm{n}^{\circ} 2,4,6,7$ et 10 n'ont pas pratiqué cette méthode. $(* \mathrm{C}, * * \mathrm{C}$ : tests de Cochran, respectivement, valeur isolée conservée ou aberrante écartée. Lorsque les deux répétitions sont aberrantes, les moyennes ne sont pas conservées pour l'analyse statistique.* $\mathrm{G}$, **G : tests de Grubbs, respectivement, valeur isolée conservée ou aberrante écartée).

supérieures, respectivement : de 0,05 à $0,19 \mathrm{~g} / 100 \mathrm{~g}$ MS pour les tourteaux déshuilés et de 0,12 à $0,16 \mathrm{~g} / 100 \mathrm{~g}$ MS pour les autres échantillons, excepté pour l'échantillon $n^{\circ} 8$ (tourteau gras lin), lequel donne un écart type de répétabilité nettement plus important avec la méthode B (0,46 g/100 g MS).

\subsection{Reproductibilité}

Les écarts-types de reproductibilité $\left(s_{R}\right)$ mesurés sur les échantillons de tourteaux déshuilés sont compris entre 0,15 (tourteau de tournesol avec méthode A) et 0,36 g/100 g MS (tourteau de colza avec méthode B) (Tab. 7). En comparaison, des valeurs de $s_{R}$ mesurées dans l'essai inter-laboratoires effectué pour la validation de la norme NF EN ISO 734-1 (méthode de référence) varient de 0,20 à $0,38 \mathrm{~g} / 100 \mathrm{~g} \mathrm{MS}$. Les écarts-types de reproductibilité mesurés sur les échantillons $\mathrm{n}^{\circ} 6$ (tourteau colza gras), $\mathrm{n}^{\circ} 7$ (graine soja extrudé) et $\mathrm{n}^{\circ} 8$ (tourteau lin gras) qui ont une teneur en huile nettement plus élevée (de 17 à $20 \mathrm{~g} / 100 \mathrm{~g} \mathrm{MS}$ ), sont légèrement supérieurs pour les méthodes $\mathrm{A}$ et $\mathrm{C}$ (de 0,20 à $0,53 \mathrm{~g} / 100 \mathrm{~g} \mathrm{MS}$ ) et nettement plus élevés pour la méthode $\mathrm{B}$ (de 0,65 à 1,04 g/100 g MS), surtout dans le cas du soja extrudé. Sur l'ensemble des échantillons, les trois types de méthodes ont de bonnes performances de reproductibilité. Le fait que les méthodes de type $\mathrm{C}$ aient des modes opératoires différents n'a pas eu d'incidence sur leur reproductibilité. 
Tableau 7. Résultats de fidélité obtenus sur les huit échantillons avec les trois types de méthodes d'extraction (méthode A : extraction au solvant sans hydrolyse ; méthode B : hydrolyse et extraction au solvant ; méthode C : extraction au solvant avec étape de broyage). Les moyennes, les écart-types de répétabilité $\left(s_{r}\right)$ et de reproductibilités $\left(s_{R}\right)$ sont exprimés en g/100 g. $C V r$ et $C V_{R}$ sont les coefficients de variation de répétabilité et de reproductibilité.

\begin{tabular}{|c|c|c|c|c|c|c|c|c|c|}
\hline Méthodes & Échantillon & $\begin{array}{c}\text { Tourteau } \\
\text { déshuilé } \\
\text { colza }\end{array}$ & $\begin{array}{c}\text { Tourteau } \\
\text { déshuilé } \\
\text { colza }\end{array}$ & $\begin{array}{c}\text { Tourteau } \\
\text { déshuilé } \\
\text { colza }\end{array}$ & $\begin{array}{l}\text { Tourteau } \\
\text { déshuilé } \\
\text { tournesol }\end{array}$ & $\begin{array}{c}\text { Tourteau } \\
\text { déshuilé } \\
\text { soja }\end{array}$ & $\begin{array}{c}\text { Tourteau } \\
\text { gras } \\
\text { colza }\end{array}$ & $\begin{array}{c}\text { Graine } \\
\text { soja } \\
\text { extrudée }\end{array}$ & $\begin{array}{c}\text { Tourteau } \\
\text { gras } \\
\text { lin }\end{array}$ \\
\hline & $\mathrm{N}^{\circ}$ & 1 & 2 & 3 & 4 & 5 & 6 & 7 & 8 \\
\hline \multirow{6}{*}{$\begin{array}{c}\text { A } \\
\text { Extraction } \\
\text { au solvant }\end{array}$} & Nombre de labos conservés & 10 & 10 & 10 & 9 & 10 & 10 & 9 & 10 \\
\hline & Moyenne & 1,869 & 3,742 & 2,745 & 1,442 & 2,275 & 17,352 & 18,582 & 18,880 \\
\hline & $s_{r}$ & 0,051 & 0,053 & 0,053 & 0,067 & 0,048 & 0,119 & 0,152 & 0,147 \\
\hline & $C V_{r}$ & $2,7 \%$ & $1,4 \%$ & $1,9 \%$ & $4,7 \%$ & $2,1 \%$ & $0,7 \%$ & $0,8 \%$ & $0,8 \%$ \\
\hline & $s_{R}$ & 0,260 & 0,238 & 0,339 & 0,146 & 0,327 & 0,308 & 0,200 & 0,372 \\
\hline & $C V_{R}$ & $13,9 \%$ & $6,4 \%$ & $12,4 \%$ & $10,2 \%$ & $14,4 \%$ & $1,8 \%$ & $1,1 \%$ & $2,0 \%$ \\
\hline \multirow{6}{*}{$\begin{array}{c}\text { B } \\
\text { Extraction au } \\
\text { solvant avec } \\
\text { hydrolyse }\end{array}$} & Nombre de labos conservés & 9 & 9 & 8 & 9 & 9 & 9 & 9 & 9 \\
\hline & Moyenne & 3,277 & 4,825 & 4,178 & 2,366 & 2,949 & 17,487 & 20,764 & 18,985 \\
\hline & $s_{r}$ & 0,185 & 0,073 & 0,098 & 0,067 & 0,056 & 0,267 & 0,126 & 0,456 \\
\hline & $C V_{r}$ & $5,6 \%$ & $1,5 \%$ & $2,3 \%$ & $2,8 \%$ & $1,9 \%$ & $1,5 \%$ & $0,6 \%$ & $2,4 \%$ \\
\hline & $s_{R}$ & 0,358 & 0,213 & 0,197 & 0,176 & 0,167 & 0,729 & 1,043 & 0,654 \\
\hline & $C V_{R}$ & $10,9 \%$ & $4,4 \%$ & $4,7 \%$ & $7,4 \%$ & $5,7 \%$ & $4,2 \%$ & $5,0 \%$ & $3,4 \%$ \\
\hline \multirow{6}{*}{$\begin{array}{c}\text { C } \\
\text { Extraction } \\
\text { au solvant } \\
\text { avec broyage }\end{array}$} & Nombre de labos conservés & 6 & 6 & 7 & 7 & 6 & 7 & 7 & 5 \\
\hline & Moyenne & 2,433 & 4,266 & 3,384 & 1,662 & 2,640 & 17,867 & 20,038 & 19,211 \\
\hline & $s_{r}$ & 0,150 & 0,124 & 0,154 & 0,168 & 0,199 & 0,159 & 0,148 & 0,142 \\
\hline & $C V_{r}$ & $6,2 \%$ & $2,9 \%$ & $4,5 \%$ & $10,1 \%$ & $7,5 \%$ & $0,9 \%$ & $0,7 \%$ & $0,7 \%$ \\
\hline & $s_{R}$ & 0,332 & 0,238 & 0,232 & 0,168 & 0,283 & 0,313 & 0,534 & 0,142 \\
\hline & $C V_{R}$ & $13,6 \%$ & $5,6 \%$ & $6,8 \%$ & $10,1 \%$ & $10,7 \%$ & $1,8 \%$ & $2,7 \%$ & $0,7 \%$ \\
\hline
\end{tabular}

Tableau 8. Écarts entre les résultats obtenus sur les 8 échantillons par les trois méthodes (méthode A : extraction au solvant; méthode B : extraction avec hydrolyse préalable; méthode $\mathrm{C}$ : extraction avec ou par broyage). Les valeurs de probabilité critique de différence entre les méthodes sont calculées à partir des lois normales et des caractéristiques de reproductibilité des méthodes $\left(s_{R}\right.$ dans Tab. 4).

\begin{tabular}{|c|c|c|c|c|c|c|c|c|c|}
\hline & & $\begin{array}{c}\text { Tourteau } \\
\text { déshuilé } \\
\text { colza } \\
1\end{array}$ & $\begin{array}{c}\text { Tourteau } \\
\text { déshuilé } \\
\text { colza } \\
2\end{array}$ & $\begin{array}{c}\text { Tourteau } \\
\text { déshuilé } \\
\text { colza } \\
3\end{array}$ & $\begin{array}{c}\text { Tourteau } \\
\text { déshuilé } \\
\text { tournesol } \\
4\end{array}$ & $\begin{array}{l}\text { Tourteau } \\
\text { déshuilé } \\
\text { soja } \\
5\end{array}$ & $\begin{array}{c}\text { Tourteau } \\
\text { gras } \\
\text { colza } \\
6\end{array}$ & $\begin{array}{c}\text { Graine } \\
\text { soja } \\
\text { extrudé } \\
7\end{array}$ & $\begin{array}{c}\text { Tourteau } \\
\text { gras } \\
\text { lin } \\
8\end{array}$ \\
\hline \multirow{2}{*}{ B-A } & Écart absolu & 1,41 & 1,08 & 1,43 & 0,92 & 0,67 & 0,14 & 2,18 & 0,10 \\
\hline & Probabilité $\mathrm{B}>\mathrm{A}$ & $99,9 \%$ & $100,0 \%$ & $100,0 \%$ & $100,0 \%$ & $97,4 \%$ & $54,3 \%$ & $98,7 \%$ & $52,7 \%$ \\
\hline \multirow{2}{*}{ C-A } & Écart absolu & 0,56 & 0,52 & 0,64 & 0,22 & 0,37 & 0,52 & 1,46 & $\mathbf{0 , 3 3}$ \\
\hline & Probabilité $\mathrm{C}>\mathrm{A}$ & $90,2 \%$ & $93,7 \%$ & $93,0 \%$ & $83,1 \%$ & $80,8 \%$ & $84,6 \%$ & $99,3 \%$ & $76,0 \%$ \\
\hline \multirow{2}{*}{ B-C } & Écart absolu & 0,84 & 0,56 & $\mathbf{0 , 7 9}$ & 0,70 & $\mathbf{0 , 3 1}$ & $-0,38$ & 0,73 & $-0,23$ \\
\hline & Probabilité $\mathrm{B}>\mathrm{C}$ & $95,9 \%$ & $96,1 \%$ & $99,7 \%$ & $99,9 \%$ & $83,1 \%$ & $33,3 \%$ & $76,7 \%$ & $39,3 \%$ \\
\hline
\end{tabular}

\subsection{Valeurs moyennes}

Le Tableau 8 compare les résultats moyens obtenus par les trois méthodes et évalue la probabilité critique $(P r)$ de l'écart entre les méthodes deux à deux. L'écart entre les méthodes $\mathrm{B}$ et A due à l'étape d'hydrolyse varie de 0,7 à 1,4 g/100 g MS pour les tourteaux déshuilés $(\operatorname{Pr}>97 \%)$. Il est plus important $(2,2 \mathrm{~g} / 100 \mathrm{~g}$ MS $)$ avec le soja extrudé $(\operatorname{Pr}>98 \%)$ et inexistant avec les tourteaux de pression de colza et lin.

L'écart entre les méthodes $\mathrm{C}$ et $\mathrm{A}$ dû à l'étape de broyage spécifique, varie de 0,5 à $0,6 \mathrm{~g} / 100 \mathrm{~g} \mathrm{MS}(\mathrm{Pr}>90 \%)$ pour les tourteaux de colza et est plus faible (0,2 à 0,4 g/100 g MS) pour les tourteaux de tournesol et soja $(\mathrm{Pr}>80 \%)$. L'écart est important avec le soja extrudé (1,5 g/100 g MS) et faible avec les tourteaux gras de colza et lin (0,3 à 0,5 g/100 g MS), surtout par rapport à la teneur en MG de ces produits (de 18 à $20 \mathrm{~g} / 100 \mathrm{~g}$ MS).

La méthode $\mathrm{B}$ donne des résultats supérieurs de 0,5 à $0,8 \mathrm{~g} / 100 \mathrm{~g} \mathrm{MS}(\mathrm{Pr}>95 \%)$ à la méthode $\mathrm{C}$ sur les tourteaux déshuilés de colza et tournesol et le soja extrudé. L'écart sur le tourteau déshuilé de soja est plus faible $(0,3 \mathrm{~g} / 100 \mathrm{~g}$ MS, $\operatorname{Pr}>80 \%$ ) et inexistant pour les tourteaux gras de colza et de lin. 


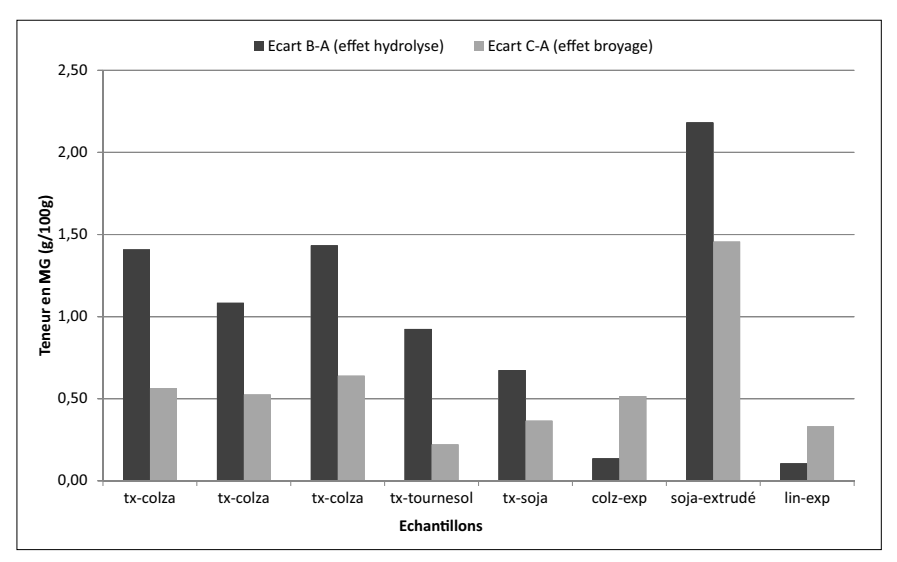

Fig. 1. Écarts moyens entre la méthode A (extraction au solvant) et les méthodes B (hydrolyse préalable) ou C (broyage) obtenus pour les teneurs en MG résiduelle des 8 échantillons.

\section{Discussion}

L'objectif de cet essai étant la comparaison des méthodes, il était important de vérifier la bonne mise en œuvre par les laboratoires et la conformité des performances par rapport aux exigences mentionnées dans les normes. Les mesures de la répétabilité et de la reproductibilité étaient aussi nécessaires pour évaluer la significativité des écarts mesurés entre les méthodes, notamment lorsqu'ils sont faibles. Bien qu'elles soient acquises avec des méthodes ayant des modes opératoires non identiques, les méthodes de type $\mathrm{C}$ se sont avérées comme les méthodes $\mathrm{A}$ et $\mathrm{B}$, répétables et reproductibles. Le nombre de laboratoires validés pour cette méthode était inférieur à huit, chiffre normalement exigé dans un circuit inter-laboratoires mais le calcul des probabilités critiques a montré dans la plupart des cas, le caractère significatif des écarts entre les méthodes testées.

Les écarts relevés avec les trois types de méthodes d'extraction au solvant (méthode $\mathrm{A}$ : de base; méthode $\mathrm{B}$ : avec hydrolyse préalable; méthode $\mathrm{C}$ : avec broyage) étaient attendus dans la mesure où la méthode $A$ n'extrait que la matière grasse libre alors que la méthode $\mathrm{B}$ extrait en plus, la matière grasse liée libérée par l'hydrolyse. La méthode C, quant à elle, extrait grâce au broyage plus fin la matière grasse non directement accessible au solvant. Ces écarts sont facilement expliqués par la nature et le traitement des échantillons. Les tourteaux de colza industriels contiennent une fraction de matière grasse résiduelle non extractible sans hydrolyse, car liée à la matrice en raison du traitement thermique important subi pendant la désolvantation. Cette fraction qui correspond à l'écart (B-A) peut être estimée à environ $1,3 \mathrm{~g} / 100 \mathrm{~g}$ MS pour le tourteau de colza, et seulement de 0,7 à $0,9 \mathrm{~g} / 100 \mathrm{~g}$ MS pour les tourteaux de soja et de tournesol; ces derniers étant plus faciles à désolvanter subissent un traitement thermique atténué (Fig. 1). Pour ces trois types de tourteaux, la fraction de matière grasse liée représente environ la moitié de la matière grasse extraite par la méthode $\mathrm{A}$. Le traitement d'extrusion à haute température justifie également l'écart de 2,2 g/100 g MS observé pour la graine de soja. Inversement, lorsque les traitements thermiques appliqués sont peu intenses, ce qui est le cas des tourteaux gras, les écarts relevés entre les méthodes B et $\mathrm{A}$ sont très faibles. Cette dernière observation ne doit pas être généralisée car il a été montré que, dans certaines conditions de conservation, ces produits peuvent contenir une fraction importante de matière grasse acidifiée et oxydée, susceptible d'échapper à la quantification par la méthode de type A (Quinsac et al. 2009). Globalement, les résultats de cette étude confirment les écarts entre les méthodes B et A reportés précédemment par Morand-Fehr et Tran (2001) pour les tourteaux de colza (1,2 g/100 g MS), de soja (0,4 g/100 g MS) et la graine de soja extrudée (2,5 g/100 g MS).

La MG non liée et non extractible directement par le solvant correspond au « défaut de broyage » car cette fraction résulte d'un accès difficile du solvant due à la persistance de barrières cellulaires ou à une structure pas assez homogénéisée par les traitements thermiques et mécaniques du procédé (aplatissage et cuisson) avant l'extraction par pressage et par le solvant. Cette fraction peut être associée à l'écart entre les méthodes $\mathrm{C}$ et $\mathrm{A}$, puisque la méthode $\mathrm{C}$ met en œuvre une étape de broyage poussé (micro-broyeur à bille) pour améliorer le contact entre la MG et le solvant. Cet écart est estimé à environ $0,5-0,6 \mathrm{~g} / 100 \mathrm{~g}$ MS pour les tourteaux déshuilés et gras de colza et est plus faible pour le tournesol et le soja, ce qui peut être rapproché du comportement différencié de ces graines observé dans les usines de trituration, aussi bien pour le rendement de pressage que pour l'extractibilité par le solvant. Cette fraction est importante dans le cas des graines de soja extrudées n'ayant pas subi de pressage.

Ces résultats sur la comparaison des méthodes montrent que les écarts constatés entre l'enquête ONIDOL-CETIOM et les tables INRA (2004) sont dus aux principes d'extraction de la matière grasse. La méthode utilisée pour l'enquête ONIDOL-CETIOM fait appel à une étape de broyage (microbroyeur à billes) et doit être qualifiée de type $\mathrm{C}$, alors que les données des tables INRA (2004) ont été obtenues par une méthode de type A, procédant par extraction au solvant sans hydrolyse préalable (Tab. 3). On peut noter d' ailleurs que l'utilisation de la méthode de type A pour les tourteaux contrevient aux recommandations relatives aux méthodes pour aliments des animaux. En effet, la directive 71/393/CEE modifiée par le directive 98/64/CE du 3/09/1998 (devenue R152/2009) spécifie qu'une hydrolyse devrait être réalisée pour «les aliments dont les matières grasses ne sont pas complètement extractibles sans hydrolyse préalable, par exemple les glutens, les levures, les protéines de pomme de terre et les aliments soumis à des procédés tels que l'extrusion, la floconnisation et le chauffage ». Bien que ne possédant pas de glycolipides comme les céréales, le tourteau de colza se situe bien dans ce dernier cas de figure car il subit des traitements hydrothermiques notables pour assurer l'élimination du solvant d'extraction. Or, il a été en fait souvent analysé par les fabricants d'aliment et les triturateurs comme un «aliment simple» pour des raisons pratiques (méthode plus simple), historique et commerciale, la même méthode étant utilisée par les deux parties contractantes lors des transactions.

Les performances différentes des trois méthodes A, B et C ont des conséquences pour la valorisation des tourteaux d'oléagineux car la sous-estimation de la teneur en MG résiduelle de ces matières premières constitue un inconvénient pour le 
fabricant et l'utilisateur. Pendant la trituration, la méconnaissance de la valeur réelle du résidu de MG restant à extraire dans le tourteau empêchera l'optimisation de l'extraction. La marge nette de trituration risque de se dégrader par les effets conjoints de la diminution de la marge brute (valeur des produits de la trituration, soit huile et tourteaux, diminuée du coût des graines triturées) et de l'augmentation du coût de la trituration. Il faut souligner que l'industrie de la trituration a déjà intégré cette problématique dans la gestion technique des usines et les investissements. Depuis 2006 et jusqu'en 2011, le suivi régulier et précis de la teneur en MG des tourteaux réalisé au cours de l'enquête CETIOM-ONIDOL, a incité les laboratoires d'usines à pratiquer des méthodes de type $\mathrm{C}$ et à partir de 2007, de nouveaux équipements d'extraction continue plus performants ont entraîné une baisse régulière de la teneur en MG résiduelle des tourteaux (de 3,6 à 2,9 g /100 g pour le colza) (Dauguet et al. 2011). Cet écart de 0,7 point représente environ $3,8 \mathrm{~kg}$ d'huile extraite en plus par tonne de graine de colza. Suivant les hypothèses de prix des produits (huile : $900 € / \mathrm{t}$, tourteau : $300 € / \mathrm{t}$ ) et de coût de trituration (environ $30 € / \mathrm{t}$ ), ce gain d'huile améliore la marge brute d'environ $2,2 €$ par tonne triturée, ce qui représente environ $7 \%$ du coût de la trituration du colza.

Dans le secteur de la fabrication des aliments pour animaux, la teneur résiduelle de la MG dans les tourteaux est devenue un élément important pour l'élaboration des formules au moindre coût, en raison de sa forte valeur énergétique et économique. Compte-tenu des traitements hydrothermiques appliqués aux tourteaux pendant leur fabrication et qui contribuent à créer une fraction de MG liée à la matrice, les méthodes « aliments pour animaux » avec hydrolyse (de type B) semblent les mieux adaptées. Cependant, la plupart des équations utilisées pour calculer les valeurs d'énergie digestible, métabolisable ou nette ont été établies à partir de données obtenues par les méthodes de type A, ce qui compense la sous-évaluation de la MG réalisée en pratique par cette méthode, et aboutit finalement à une évaluation de l'énergie assez juste (INRA, 2004). Sur des lots de tourteau un peu atypiques (fortement chauffés, un peu gras, etc.), il n'en reste pas moins possible que la MG non extraite par la méthode de type A soit assez différente de la valeur habituelle et dégrade la précision du modèle de prédiction.

\section{Conclusion}

Les trois méthodes testées et utilisées par les laboratoires dans cet essai inter-laboratoires se sont révélées robustes et reproductibles. L'essai mené sur les huit échantillons a pu montrer que ces méthodes évaluaient différemment les teneurs en MG résiduelle en raison de la présence ou non d'étapes d'hydrolyse et de broyage spécifique. Par rapport à la méthode de base (A : extraction par solvant), les méthodes avec broyage (C) extraient davantage de $\mathrm{MG}(+0,2$ à $0,7 \mathrm{~g} / 100 \mathrm{~g} \mathrm{MS})$, et les méthodes avec hydrolyse, encore plus (+0,6 à 1,4 g/100 g MS). Ces caractéristiques doivent être connues des utilisateurs des méthodes pour d'une part, mieux exploiter les données existantes et d'autre part, mieux choisir les méthodes d'analyses, en fonction de leurs besoins propres.

De manière générale, il convient de recommander pour le dosage de la matière grasse résiduelle des tourteaux d'oléagineux, les méthodes qui leur sont dédiées et décrites dans les normes NF EN ISO 734-1 (méthode de référence) ou NF EN ISO 734-2 (méthode d'extraction rapide). Pour l'alimentation animale, la méthode dédiée avec hydrolyse préalable (NF V18-117 partie B, directive 71/393/CEE partie B, modifiée par la directive 98/64/CE partie B) doit être utilisée en gardant à l'esprit qu'une partie de la MG mesurée risque de ne pas être valorisée de manière optimale. Lorsque des équations de prédiction de l'énergie sont utilisées, les données à traiter doivent en principe être obtenues avec même type de méthode (le plus souvent avec NF V18-117 partie A, directive 71/393/CEE partie A, modifiée par la directive 98/64/CE partie A).

Les résultats de cet essai inter-laboratoires et ces recommandations devraient prochainement faire l'objet d'un document normatif publié par l'AFNOR.

Remerciements. Les auteurs remercient Jean Noblet de UMR PEGASE, INRA Saint-Gilles (35) pour le partage de son expertise et sa contribution à ce travail. Ils remercient également les sociétés DIALZO, COACHE, SAIPOL-Dieppe et SAIPOL-Compiègne pour la fourniture des lots de graines de soja extrudé, de tourteaux de lin et de colza.

\section{Références}

AFNOR. 1988. V03-908. Juin 1988. Graines oléagineuses. Détermination de la teneur en huile. Méthode simplifiée par extraction à l'hexane.

Dauguet S, Crépon K, Loison JP. 2006. Enquêtes sur la qualité nutritionnelle des tourteaux industriels. Oléoscope 87: 4-6.

Dauguet S, Krouti M, Loison JP, Peyronnet C. Quinsac A. A multiyear survey on the chemical composition of rapeseed meal produced in France. In : Proc. 13th Intl. Rapeseed Congress, 2011, Prague. Ed. SPZO Prague, ISBN 978-87065-33-4.

INRA. 2004. Tables de composition et de valeur nutritive des matières premières destinées aux animaux d'élevage. Paris : INRA Editions.

JOCE. 1998. Directive 98/64/CE de la Commission du 3 septembre 1998 portant fixation des méthodes d'analyse communautaires pour la détermination des acides aminés, des matières grasses brutes et de l'olaquindox dans les aliments des animaux et modifiant la directive 71/393/CEE. Journal officiel $n^{\circ}$ L $257 \mathrm{du}$ 19/09/1998. 0014-0028.

JOUE. 2009. Règlement (CE) $N^{\circ} 152 / 2009$ de la Commission du 27 janvier 2009 portant fixation des méthodes d'échantillonnage et d'analyse destinées au contrôle officiel des aliments pour animaux - Méthodes d'analyse relative au contrôle de la composition des matières premières pour aliments des animaux et des aliments composés. Annexe III, point H : Dosage des Matières grasses brutes. Journal officiel de l'Union Européenne du 26.2.2009. L54/37-42.

Laisney J. 1984. Le traitement des graines oléagineuses. In : Compagnie Française pour le développement des fibres textiles. Paris : L'huilerie moderne.

Morand-Fehr P, Tran G. 2001. La fraction lipidique des aliments et les corps gras utilisés en alimentation animale. INRA Prod. Anim. 14: 285-302. 
NF EN ISO 659. Septembre 2009. Graines oléagineuses. Détermination de la teneur en huile. Méthode de référence.

NF EN ISO 734-1. Janvier 2007. Tourteaux de graines oléagineuses. Détermination de la teneur en huile. Partie 1 : Méthode par extraction à l'hexane (ou à l'éther de pétrole).

NF EN ISO 734-2. Juillet 2008. Tourteaux de graines oléagineuses. Détermination de la teneur en huile. Partie 2 : Méthode rapide par extraction.

NF EN ISO 734-2. Juillet 2010. Tourteaux de graines oléagineuses. Détermination de la teneur en huile. Partie 2 : Méthode rapide par extraction.

NF ISO 5725-2. Décembre 1994. Application de la statistique. Exactitude (justesse et fidélité) des résultats et méthodes de mesure.
NF ISO 6492. Mars 2011. Aliments des animaux. Détermination de la teneur en matière grasse.

NF V03-908. Décembre 2009. Graines oléagineuses. Détermination de la teneur en huile. Méthode alternative.

NF V18-117. Août 1997. Aliments des animaux. Dosage de la matière grasse.

Quinsac A. Carré P, Crépon K, Evrard J, Loison J-P, Lessire M, Peyronnet C. 2009. Characteristics of the expeller rapeseed meal production in farms and industrial plants in France. Assessment of the meal stability in long-term storage and the energetic value on poultry. In : Proc. 28th Intl Soc. Fat Res. Congress, September, 28-30, 2009, Sydney.

Woonacott TH, Woonacott RJ. 1988. Statistique. Economie - Gestion - Sciences - Médecine. Paris : Economica.

Cite this article as: Alain Quinsac, Charles-Pierre Bazin de Caix, Claude Bernard, Brigitte Brosson, Patrick Carre, Gwenaëlle Chardon, Michel Chapron, Danièle Chataignier, Christophe Ferey, Clémence Gaucher, Christophe Genouel, Mohammed Krouti, Renaud Le Bouquin, Jean-Philippe Loison, Hugues Marescot, Valérie Salvador, Ludovic Sarcher, Nathalie Scorze, Danièle Simonneau. Comparaison des méthodes de détermination de la matière grasse dans les tourteaux d'oléagineux - Étude collaborative inter-laboratoires. OCL 2013, 20(5) A503. 